\title{
The p90 ribosomal S6 kinase (RSK) inhibitor BI-D1870 prevents gamma irradiation-induced apoptosis and mediates senescence via RSK- and p53-independent accumulation of $\mathrm{p} 21^{\mathrm{WAF} 1 / \mathrm{CIP} 1}$
}

\author{
D Neise ${ }^{1}$, D Sohn ${ }^{1}$, A Stefanski ${ }^{2}$, H Goto $^{3}$, M Inagaki ${ }^{3}$, S Wesselborg ${ }^{4}$, W Budach ${ }^{1}, K_{\text {Stühler }}^{2}$ and RU Jänicke ${ }^{\star, 1}$
}

The 990 ribosomal $\mathbf{S 6}$ kinase (RSK) family is a group of highly conserved Ser/Thr kinases that promote cell proliferation, growth, motility and survival. As they are almost exclusively activated downstream of extracellular signal-regulated kinases 1 and 2 (ERK1/2), therapeutic intervention by RSK inhibition is less likely to produce such severe side effects as those observed following inhibition of the upstream master regulators Raf, MEK and ERK1/2. Here, we report that BI-D1870, a potent small molecule inhibitor of RSKs, induces apoptosis, although preferentially, in a p21-deficient background. On the other hand, BI-D1870 also induces a strong transcription- and p53-independent accumulation of p21 protein and protects cells from gamma irradiation $(\gamma \mid \mathrm{I})$-induced apoptosis, driving them into senescence even in the absence of $\gamma \mathrm{IR}$. Although we identified p21 in in vitro kinase assays as a novel RSK substrate that specifically becomes phosphorylated by RSK1-3 at Ser116 and Ser146, RNA-interference, overexpression and co-immunoprecipitation studies as well as the use of SL0101, another specific RSK inhibitor, revealed that BI-D1870 mediates p21 accumulation via a yet unknown pathway that, besides its off-site targets polo-like kinase- 1 and AuroraB, also does also not involve RSKs. Thus, this novel off-target effect of BI-D1870 should be taken into serious consideration in future studies investigating the role of RSKs in cellular signaling and tumorigenesis.

Cell Death and Disease (2013) 4, e859; doi:10.1038/cddis.2013.386; published online 17 October 2013

Subject Category: Cancer

Ribosomal S6 kinases (RSKs) constitute a family of serine/ threonine kinases that in response to various stimuli are almost exclusively activated by extracellular signal-regulated kinases 1/2 (ERK1/2). ${ }^{1}$ Thus, RSKs are downstream effectors of the Ras/mitogen-activated protein kinase (MAPK) pathway that is crucial for normal cell proliferation, differentiation, motility and survival. The importance of this pathway is emphasized by the fact that mutations or overexpression of many of the involved signaling components are hallmarks of several human cancers. ${ }^{2}$ The RSK family consists of four isoforms (RSK1-4) that are products of separate genes sharing a high degree of sequence homology (75-80\% identity). With the exception of RSK4, they are ubiquitously expressed, but are unique among kinases as they possess two distinct and functional kinase domains: the N-terminal kinase domain (NTKD) that is responsible for substrate phosphorylation and the C-terminal kinase domain (CTKD) required for RSK autoactivation. ${ }^{1}$ Activation of RSKs is achieved via a multistep phosphorylation cascade involving ERK1/2-mediated activation of the CTKD that in turn autophosphorylates a hydrophobic turn motif in RSK molecules. This creates a docking site for $3^{\prime}$-phospho-inositidedependent kinase-1 (PDK1), a constitutively active Ser/Thr kinase, which phosphorylates and activates the NTKD.

Among a long list of proteins targeted by RSKs are, for instance, the pro-survival transcription factors cyclic AMPresponsive element-binding protein-1 (CREB1) and nuclear factor $-\kappa \mathrm{B}(\mathrm{NF}-\kappa \mathrm{B})$, as well as proteins stimulating capdependent translation, including the eukaryotic translation initiation factor-4B (elF-4B) and ribosomal protein-S6 (rpS6). ${ }^{3-7}$ With the phosphorylation and inactivation of several cell cycle-regulatory and pro-apoptotic proteins such as the cyclin-dependent kinase (CDK) inhibitor p27, Chk1, BAD, BimEL, caspase-8 and p53, activated RSKs are

\footnotetext{
${ }^{1}$ Laboratory of Molecular Radiooncology, Clinic and Policlinic for Radiation Therapy and Radiooncology, University of Düsseldorf, Universitätsstrasse 1, Düsseldorf 40225, Germany; '2Molecular Proteomics Laboratory, BMFZ, University of Düsseldorf, Universitätsstrasse 1, Düsseldorf 40225, Germany; ${ }^{3}$ Aichi Cancer Center, 1-1 Kanokoden, Chikusa-ku, Nagoya, Aichi 464-8681, Japan and ${ }^{4}$ Institute of Molecular Medicine, University of Düsseldorf, Universitätsstrasse 1, Düsseldorf 40225, Germany

*Corresponding author: RU Jänicke, Laboratory of Molecular Radiooncology, Clinic and Policlinic for Radiation Therapy and Radiooncology, University of Düsseldorf, Building 23.12, Universitätsstrasse 1, Düsseldorf D-40225, Germany. Tel: +49 21181 15515; Fax: +49 21181 15892; E-mail: janicke@ uni-duesseldorf.de Keywords: apoptosis; BI-D1870; cell cycle; off-target effect; senescence; SL0101

Abbreviations: ACN, acetonitrile; AGC kinase, protein kinase A, G, C family; CDK, cyclin-dependent kinase; Chx, cycloheximide; CTKD, C-terminal kinase domain; DMSO, dimethylsulfoxide; ERK, extracellular signal-regulated kinase; $\gamma \mathrm{IR}$, gamma irradiation; GST, glutathione S-transferase; JNK, c-Jun N-terminal kinase; MALDI-MS, matrix-assisted laser desorption ionization-mass spectrometry; MAPK, mitogen-activated protein kinase; MEK, MAPK/ERK kinase; NTKD, N-terminal kinase domain; PLK-, polo-like kinase-1; PMA, phorbol-12-myristate-13-acetate; PKC, protein kinase C; RSK, p90 ribosomal S6 kinase; SA- $\beta$-Gal, senescenceassociated $\beta$-galactosidase; siRNA, small interfering RNA

Received 15.7.13; revised 16.8.13; accepted 05.9.13; Edited by G Raschellá
} 
proposed to strongly promote cell cycle progression and survival..$^{8-13}$ In line with these conclusions, it was shown that pharmacological or siRNA-mediated inhibition of RSK activity and/or expression caused a G1 cell cycle block in several tumor lines, ${ }^{14,15}$ whereas RSK2 overexpression enhanced colony formation even in the absence of tumor promoters. ${ }^{16}$ Accordingly, elevated RSK $1 / 2$ levels were found in several tissue samples from breast and prostate cancer patients compared with the corresponding normal tissues. ${ }^{14,15}$ Furthermore, dominant-negative RSK1 alleles eliminated survival that was mediated by the ERK-activating kinase MEK, ${ }^{17}$ whereas constitutively active RSK2 promoted cell survival at levels equivalent to those obtained with an activated MEK enzyme. ${ }^{10}$ Together, these data suggest that RSK $1 / 2$ are critically involved in cell proliferation and transformation, making them promising therapeutic targets for the treatment of cancer.

One of the most important regulators of cell cycle progression is the CDK inhibitor p21 that mediates DNA damage-induced cell cycle arrest mainly in a p53-dependent manner. In addition, p21 also participates in other diverse processes including transcription, DNA repair, differentiation, senescence and apoptosis. ${ }^{18,19}$ Thus, mechanisms are required that tightly control its expression and functional diversity. This is achieved, for instance, by multiple phosphorylations that either result in its stabilization or in proteasomal degradation. ${ }^{20}$ In addition, expression of p21 is also controlled at the transcriptional and post-transcriptional level, giving the cell multiple opportunities to interfere with p21 function. ${ }^{21-24}$

Here we show that treatment of cells with the small molecule RSK inhibitor BI-D1870 results in a transcriptionand p53-independent accumulation of p21, as well as in senescence induction and protection from $\gamma / \mathrm{IR}$-induced apoptosis. Although we subsequently identified p21 as a novel in vitro substrate for RSKs, numerous experiments revealed that BI-D1870 mediates these stress responses via a yet unknown RSK-independent pathway. Thus, our study uncovers novel off-target effects of BI-D1870 that should be taken into consideration in further studies involving this compound.

\section{Results}

Effect of MAPK inhibitors on radiation responses of HCT116 cell lines. Recently, we reported that p21 not only inhibits but also activates several kinases including MAPKs in a substrate-dependent manner. ${ }^{25}$ As MAPKs are critical components of cellular stress and survival pathways, ${ }^{26,27}$ we analyzed their contribution to gamma-irradiation $(\gamma \mathrm{IR})$-induced stress responses of HCT116 colon carcinoma cells. We have chosen this particular system as p21 determines therein the fate of irradiated cells. ${ }^{23,28}$ Whereas $\gamma \mathrm{IR}$ drives wild-type HCT116 cells into senescence due to p53-dependent p21 induction, similarly treated checkpointdeficient (p21 - / - and p53-/ - ) HCT116 cells succumb to apoptosis (Figures 1a and b). ${ }^{23,28}$ This is because p21 functions not only as an inducer of cellular senescence but also as a potent apoptosis inhibitor. ${ }^{19}$ Interestingly, irradiation of p53- and p21-deficient HCT116 cells in the presence of U0126, an inhibitor of the ERK1/2-activating kinases MEK1/2, protected them from apoptosis, as evidenced microscopically (not shown) and by inhibition of caspase-3-like DEVDase activity (Figure 1a). Incubation of the cells with the c-Jun N-terminal kinase (JNK) inhibitor-I or SB203580 targeting p38 had no effect on their radiation responses (Figure 1a). Moreover, although all three MAPK inhibitors did not greatly influence cell cycle distribution of unstressed and irradiated cells (Figure 1c; not shown), p53- and p21-deficient cells rescued by U0126 from $\gamma$ IR-induced apoptosis showed an enlarged and flattened morphology, exhibiting increased senescence-associated $\beta$-galactosidase (SA- $\beta$-Gal) activities (Figure 1b). Remarkably, these senescence markers were even evident when checkpoint-deficient and wild-type HCT116 cells were treated solely with U0126 (Figure 1b). As inhibition of JNK and p38 affected neither $\gamma$ IR-induced apoptosis (Figure 1a) nor senescence (not shown), our data suggest an involvement of the MEK/ERK pathway in radiation responses of HCT116 cells that is functionally independent of p53 and $\mathrm{p} 21$.

Effect of the RSK inhibitor BI-D1870 on radiation responses of HCT116 cell lines. Among a multitude of effectors targeted by ERK $1 / 2$ are RSK family members that are almost exclusively activated by these MAPKs. ${ }^{1}$ To examine their role in radiation responses of HCT116 cells, we irradiated wild-type and checkpoint-deficient cells in the absence and presence of BI-D1870, a potent RSK inhibitor. ${ }^{29}$ BI-D1870 almost completely prevented $\gamma$ IR-induced apoptosis of p53-deficient HCT116 cells (Figure 2a) and, similar to U0126, produced the senescent phenotype in both wild-type and p53-deficient cells regardless of whether or not they were irradiated (Figure 2b). BI-D1870 also induced senescence in MCF-7/casp3 breast carcinoma cells, indicating a more general response to this compound (Supplementary Figure S1). In contrast to U0126, however, BI-D1870 did not exert these effects in p21-deficient cells (Figures 2a and b), although it efficiently induced a transient G2/M arrest in all three non-irradiated HCT116 lines (Figure 2c). In addition, BI-D1870 by itself induced massive apoptosis in p21-deficient cells, whereas wild-type and p53-/- cells were killed by this compound to a much lesser extent (Figure 2a), suggesting a close functional relationship between RSKs and p21.

BI-D1870 induces transcription and p53-independent p21 accumulation. Therefore, we analyzed p21 expression in BI-D1870-treated cells and found that this compound induced a substantial p21 accumulation in HCT116 wild-type cells albeit to a lesser extent compared with $\gamma \mathrm{IR}$ (Figure 3a). In contrast, expression of p27 and p57, two other CDK inhibitors of the CIP/KIP family, remained unaffected (Figure 3b). Interestingly, BI-D1870-mediated p21 accumulation appears to occur independently of p53 that was only stabilized following $\gamma \mathrm{IR}$, but not in response to BI-D1870 (Figure 3a). Consistently, whereas p21 readily accumulated also in BI-D1870-treated p53-deficient cells $\gamma \mathrm{IR}$ failed to induce p21 expression in the absence of p53 (Figure 3c). Moreover, only $\gamma \mathrm{IR}$, but not BI-D1870 or DMSO, induced p21 mRNA expression (Figure 3d), demonstrating that 

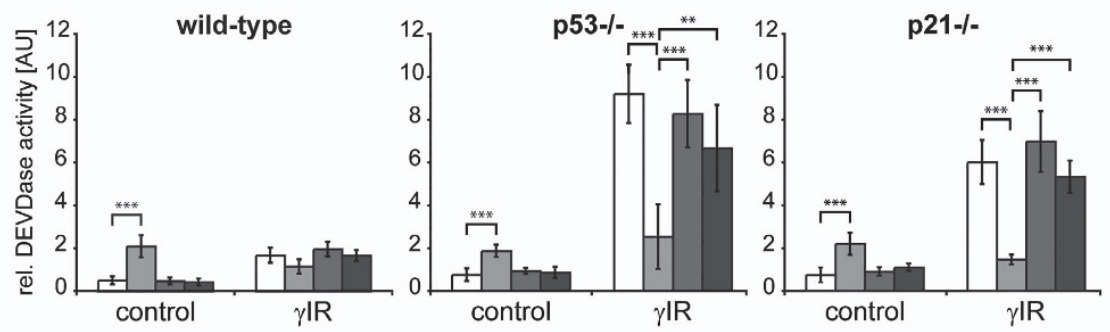

b
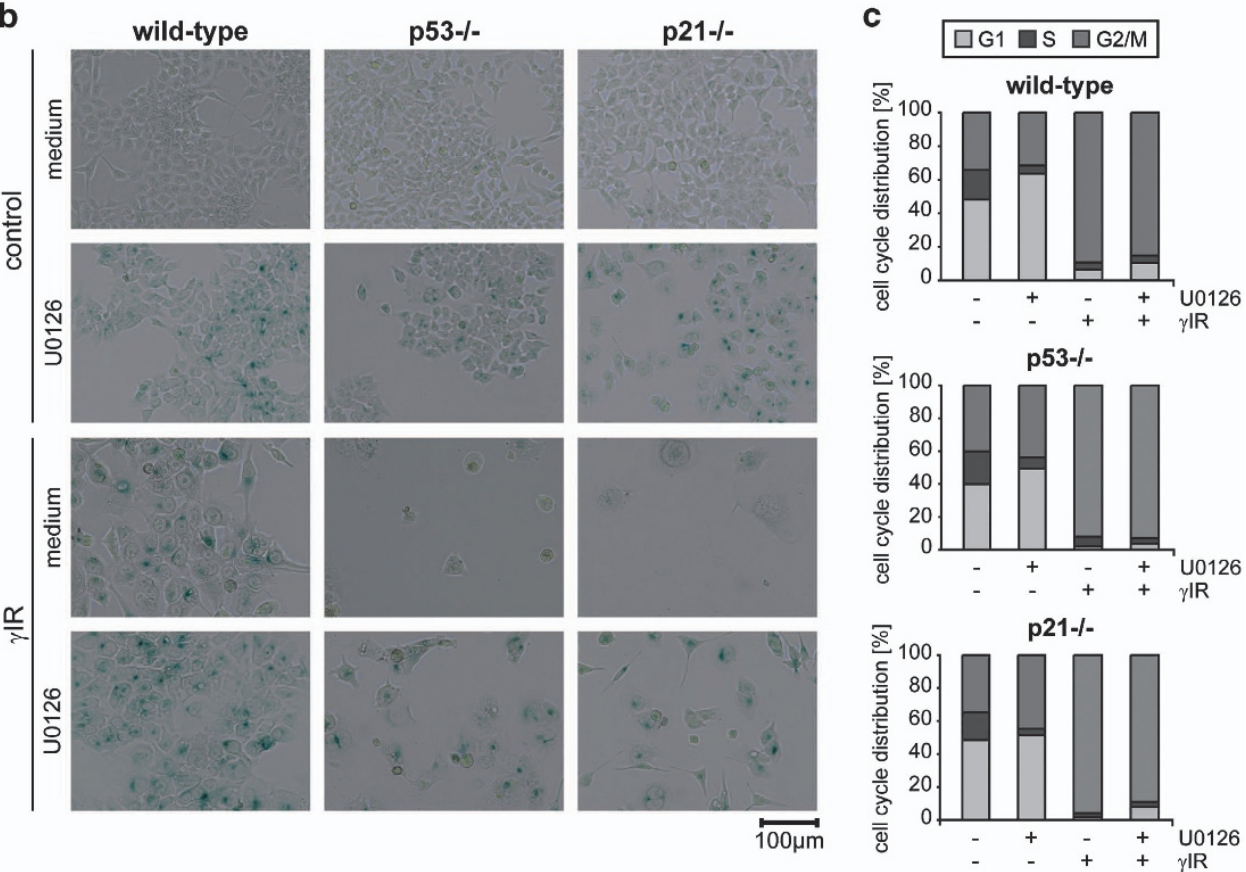

Figure 1 Effect of MAP kinase inhibitors on $\gamma / \mathrm{R}$-induced stress responses. (a) U0126 rescues checkpoint-deficient HCT116 cells from $\gamma / \mathrm{R}$-induced apoptosis independently of p21. Determination of DEVDase activities in wild-type and checkpoint-deficient (p53-/ -, p21-/ -) HCT116 cells 3 days after they were either left untreated (control) or $\gamma$-irradiated in the absence (medium) or presence of the MEK inhibitor U0126 (20 $\mu \mathrm{M})$, the JNK inhibitor-I ( $1 \mu \mathrm{M})$ or the p38 inhibitor SB203580 (20 $\mu \mathrm{M})$. Arbitrary units $(\mathrm{AU})$ shown are the mean of at least four independent experiments $+I-$ S.D. ${ }^{\star \star} P<0.01 ;{ }^{\star \star \star} P<0.005$, paired Student's $t$-test. (b) U0126 induces cellular senescence independently of p21. Determination of SA- $\beta$-Gal activity in the indicated HCT116 cell lines 4 days after they were treated as in a. One representative experiment out of three is shown. (c) Effect of U0126 on cell cycle distribution. The indicated HCT116 cells were treated as in a and the number of cells in the different cell cycle phases was determined after $24 \mathrm{~h}$ by FACS analyses. Percentages shown are the mean of three independent experiments. For a to $\mathbf{c}$, cells were preincubated with $\mathrm{U} 0126$ for 30 min and with the JNK inhibitor-I and SB203580 for $2 \mathrm{~h}$

BI-D1870-induced p21 upregulation occurs independently of p53 most likely by a post-translational event.

Consistent with our observation that BI-D1870 was able to induce senescence in MCF-7/casp3 cells as well (Supplementary Figure S1), this event was also accompanied by p21 upregulation (Figure 3e). In contrast, we did not detect an increase in p21 expression when MCF-7/casp3 or HCT116/p53 - / - cells were exposed to U0126 (Figures 3e and f), which is in agreement with our observation that U0126, unlike BI-D1870, mediates apoptosis resistance and senescence induction independently of p21 (Figures 1 and 2).

AuroraB and polo-like kinase-1 are not involved in BI-D1870-induced p21 accumulation. Although BI-D1870 was originally postulated to specifically inhibit RSKs, this and another report also demonstrated BI-D1870-mediated inhibition of polo-like kinase-1 (PLK1) and AuroraB, albeit with lower potencies. ${ }^{29,30}$ As PLK1 and AuroraB are crucial regulators of cell division that are often overexpressed in several cancers and whose inhibition elicits severe stress responses such as senescence and apoptosis induction, ${ }^{31}$ we determined their involvement in BI-D1870-mediated cellular responses. Although RNA-interference studies revealed that depletion of AuroraB or PLK1 resulted in p21 accumulation in HCT116 wild-type cells, this event was accompanied in both cases by an increase in p53 expression, indicating p53-dependent stress responses (Figures 4a and $b$, lanes 1-3). Indeed, in the absence of p53, p21 upregulation was only observed when p53-deficient HCT116 cells were treated with BI-D1870, but not in response to control, PLK1 or AuroraB siRNAs (Figure 4b, lanes 4-6; Figure 4c). In addition, silencing PLK1 resulted in massive apoptosis of HCT116 wild-type cells that was much more pronounced than in BI-D1870-treated cells (compare 
a

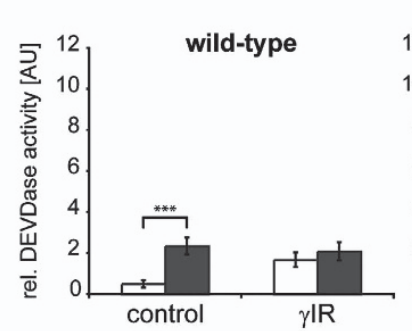

b
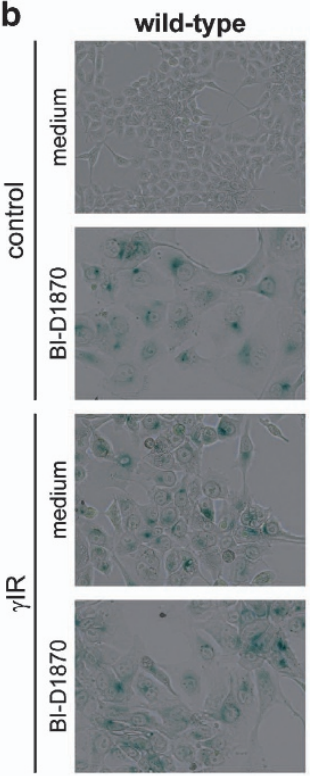
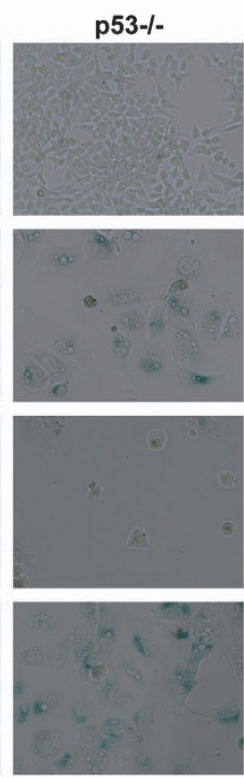

口medium घBI-D1870 (RSK)

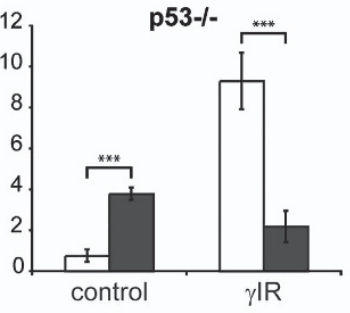

p21-/-
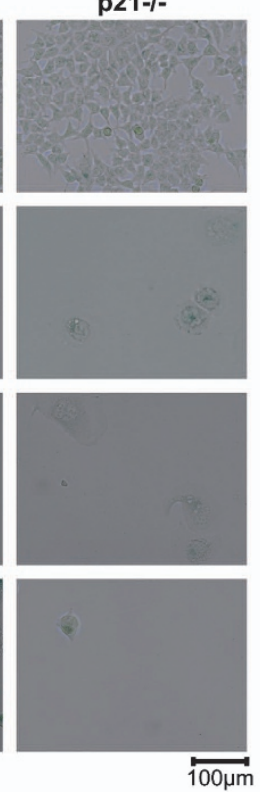

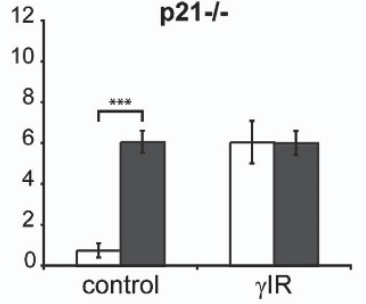

C $\square G 1 \square S \square G 2 / M$

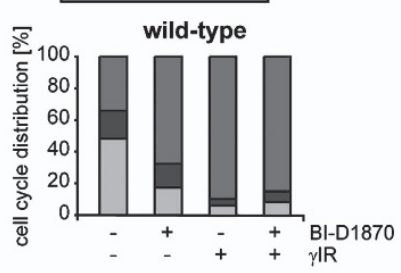

p53-/-

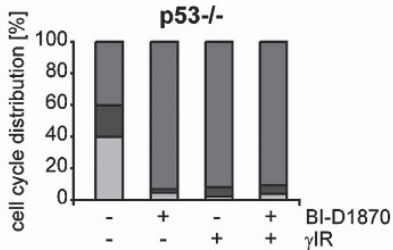

p21-/-

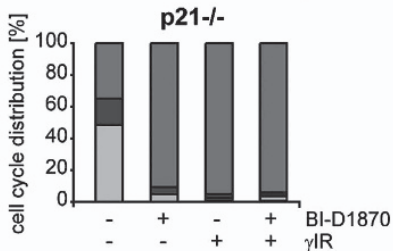

Figure 2 Effect of the RSK inhibitor BI-D1870 on $\gamma$ IR-induced stress responses. (a) BI-D1870 rescues HCT116 cells from $\gamma \mid \mathrm{IR}$-induced apoptosis in a p21-dependent manner. Determination of DEVDase activities in wild-type and checkpoint-deficient (p53 - / - , p21 - / - ) HCT116 cells 3 days after they were either left untreated (control) or $\gamma$-irradiated in the absence (medium) or presence of BI-D1870 $(10 \mu \mathrm{M})$. Arbitrary units (AU) shown are the mean of three independent experiments $+I-\mathrm{S} . \mathrm{D}$. ${ }^{* * \star} P<0.005$, paired Student's $t$-test. (b) BI-D1870 induces cellular senescence in a p21-dependent manner. Determination of SA- $\beta$-Gal activity in the indicated HCT116 cell lines 4 days after they were treated as in a. One representative experiment out of three is shown. (c) Effect of BI-D1870 on cell cycle distribution. The indicated HCT116 cells were treated as in $\mathbf{a}$, and the number of cells in the different cell cycle phases was determined after $24 \mathrm{~h}$ by FACS analyses. Percentages shown are the mean of three independent experiments. For a to c, cells were preincubated with BI-D1870 for $2 \mathrm{~h}$

Figures $2 \mathrm{a}$ and $4 \mathrm{~d})$. Together, these data demonstrate that BI-D1870-induced p21 upregulation and the consequent antiapoptotic and pro-senescent effects do not involve AuroraB or PLK1, but may depend on RSK inhibition.

RSK1-3 isoforms phosphorylate p21 in vitro at Ser116 and Ser146. As RSKs phosphorylate and inactivate several anti-apoptotic and cell cycle-regulatory proteins including p27, ${ }^{8}$ we investigated whether p21 might be targeted by RSKs. Indeed, in vitro kinase assays revealed that recombinant active RSK isoforms (RSK1/2/3) specifically phosphorylate GST-tagged p21, but not GST alone (Figures $5 a$ and b). Furthermore, BI-D1870 inhibited RSK2-mediated phosphorylation of GST-p21 in a dose-dependent manner, verifying the specificity of this reaction (Figure $5 \mathrm{c}$ ). In order to identify the RSK phosphorylation site(s) in p21, we first reasoned that RSKs might target Thr145 or Ser146, as these residues are known to be phosphorylated by Akt and PKC $\zeta$, respectively, which together with RSKs belong to the AGC kinase family sharing several structural and functional similarities. ${ }^{32}$ However, whereas the p21(T145A) mutant was not phosphorylated by Akt, it was even more intensively phosphorylated by RSK2 compared with the p21 wild-type protein (Figure $5 d$ ). An inverse picture emerged when we analyzed the p21(S146A) mutant that was phosphorylated by Akt, but hardly by RSK2 (Figure 5d). Thus, we concluded that Akt and RSK2 phosphorylate the adjacent Thr145 and Ser146 sites in p21, respectively.

In addition to Ser146, mass spectrometric analyses of in vitro RSK-phosphorylated GST-p21 also identified Ser116 as a phospho-acceptor site for RSK2 (Supplementary Figure S2), a finding that was confirmed by mutagenesis studies (Figures $5 \mathrm{c}-\mathrm{e}$ ). Although the decrease in phosphorylation of the p21(S116A) mutant was not as substantial as that observed with the p21(S146A) mutant, it was still reproducibly less phosphorylated by RSK2 than was the GST-p21 wild-type protein (Figure 5d). Consistently, an additive effect was achieved with the p21 double mutant 


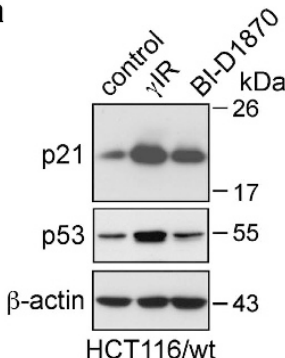

b

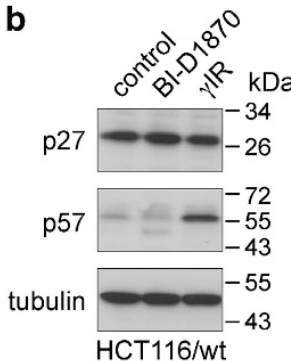

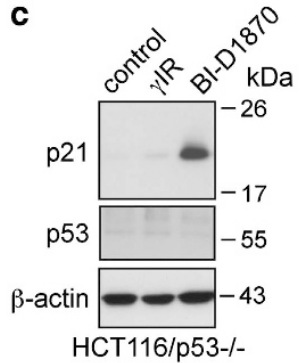

d

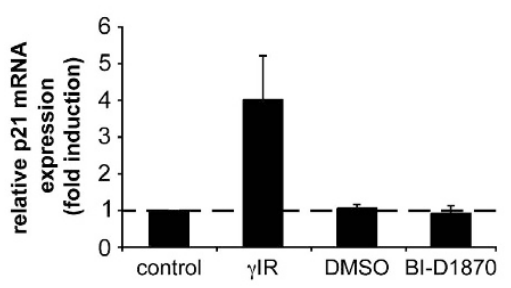

e

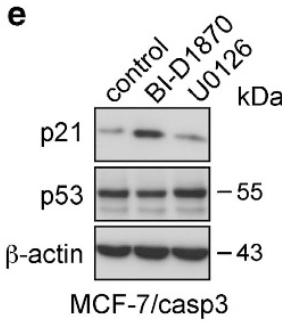

f

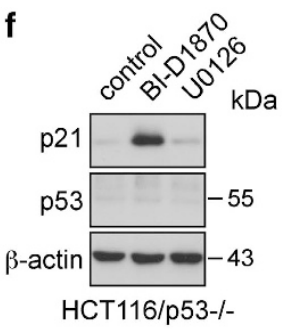

Figure 3 BI-D1870 induces p21 expression in a transcription- and p53-independent manner. (a-c and $\mathbf{e}$ and f) Western blot analyses for the status of p21, p27, p57 and p53 $24 \mathrm{~h}$ after the indicated cell lines were either left untreated (control) or exposed to $\gamma \mid \mathrm{R}, \mathrm{BI}-\mathrm{D} 1870(10 \mu \mathrm{M})$ or $\mathrm{U} 0126(20 \mu \mathrm{M})$. Blots shown are representative results of three independent experiments. (d) Real-time PCR for the determination of the expression levels of p21 mRNA in HCT116 wild-type cells that were either left untreated (control) or exposed to $\gamma \mathrm{IR}, \mathrm{BI}-\mathrm{D} 1870(10 \mu \mathrm{M})$ or DMSO $(0.1 \%)$. Total RNA was isolated $24 \mathrm{~h}$ post treatment and analyzed with transcript-specific probes from Applied Biosystems. Data shown are the mean of three independent experiments $+/-$ S.D.

a
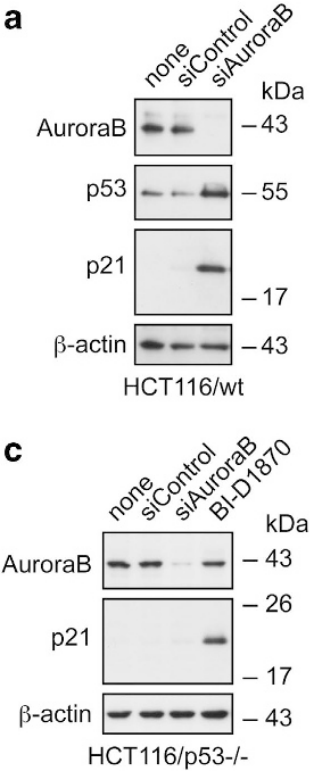

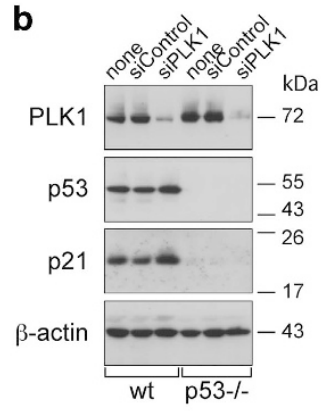

d

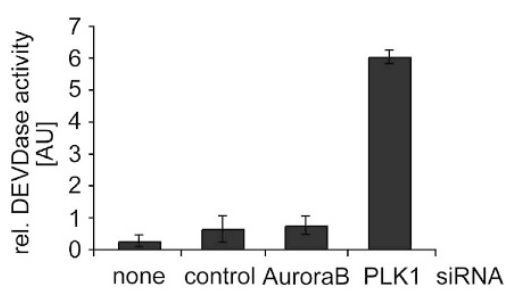

Figure 4 Effect of the knockdown of BI-D1870-inhibitable kinases on p21 expression. (a-c) Western blot analyses for the status of p21 and p53 $48 \mathrm{~h}$ after the indicated cell lines were transfected with control siRNA, AuroraB siRNA $(\mathbf{a}, \mathbf{c})$ or with PLK1 siRNA (b) or following treatment with BI-D1870 $(10 \mu \mathrm{M})(\mathbf{c})$. Please note that the $\mathrm{p} 21$ blot in $B$ is overexposed to show that $p 21$ is indeed not induced by the PLK1 siRNA in p53-deficient cells. Blots shown are representative results of three independent experiments. (d) Determination of DEVDase activity obtained $48 \mathrm{~h}$ post transfection of HCT116 wild-type cells with the indicated siRNAs. Data shown are the mean of three independent experiments $+/-$ S.D.

(S116A/S146A) that remained almost completely unphosphorylated in the presence of RSK2, verifying that both residues constitute RSK2 phospho-acceptor sites (Figure 5d). As introduction of these single and double mutations also

compromised p21 phosphorylation by RSK1/3 (Figure 5e), but not by Akt (Figure $5 \mathrm{~d}$ ), our data demonstrate that p21 is phosphorylated in vitro at Ser116 and Ser146 by all three RSKs.

In contrast to the amino acid sequence surrounding Ser146 (KRRQTpS), the residue Ser116 (VDLSLpS) does not lie within an RSK consensus sequence (BxBxxpS/pT in which $B$ is a basic aa) that is usually phosphorylated in vivo by the NTKD of RSKs. Therefore, we asked whether Ser116 may represent an artificial in vitro CTKD target that in vivo is not involved in substrate phosphorylation. We incubated the p21(S146A) mutant (in which Ser116 remains RSK phosphorylatable) with recombinant RSK2 in the presence of increasing concentrations of BI-D1870 that exclusively acts as an ATP-competitive inhibitor of the NTKD of RSKs. ${ }^{29}$ Similar to phosphorylation of wild-type p21 and the p21(S116A) mutant, the already strongly diminished RSK2mediated phosphorylation of the p21(S146A) mutant was completely blocked by BI-D1870, whereas DMSO had no effect (Figure 5c). This suggests that both the consensus site at Ser146 and that at Ser116, which is embedded in a nonconsensus sequence, are phosphorylated in vitro by the NTKD of RSKs.

RSKs are not involved in BI-D1870-induced p21 accumulation. To verify this hypothesis also in vivo, we first probed immunoprecipitated Flag-p21wt from MCF-7/ casp3 cells treated for 2 hours with PMA, a potent activator of RSKs, ${ }^{33}$ with a phospho-Ser146-specific p21 antibody. Indeed, PMA stimulation resulted in RSK activation and in a strong p21(Ser146) phosphorylation that was partially, but significantly, prevented when the cells were co-treated with BI-D1870 (Figures 6a and b). MALDI-MS analysis confirmed PMA-induced Ser146 phosphorylation of Flag-p21wt 
a

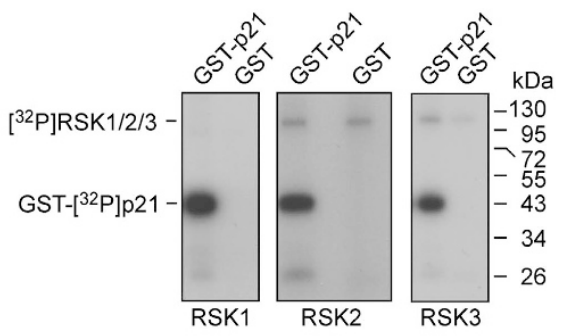

c

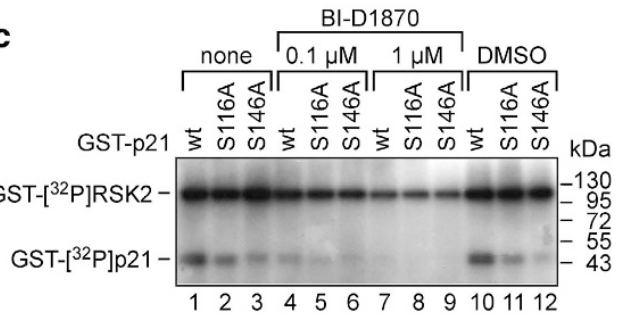

e
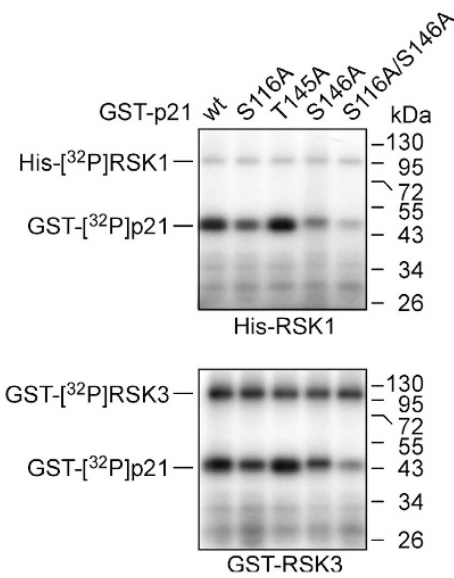

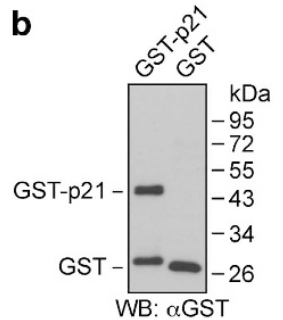

d
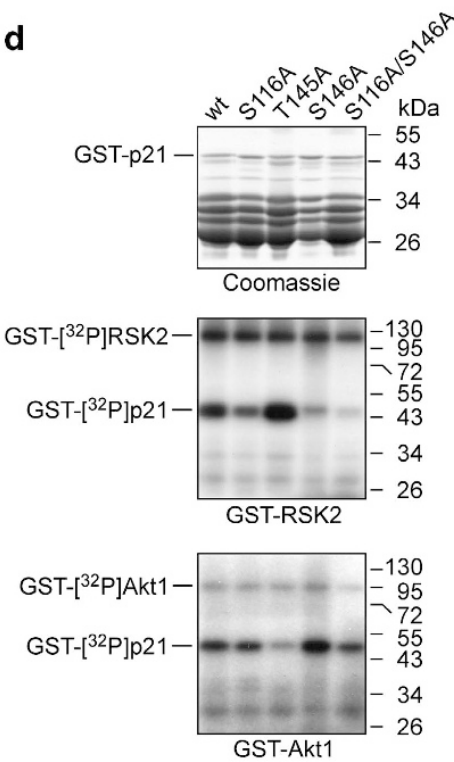

Figure 5 p21 is a novel RSK substrate in vitro. (a and $\mathbf{b})$ p21 is phosphorylated by all three RSK isoforms. Recombinant GST-p21 and GST were either incubated for the in vitro kinase assays (a) with the indicated active RSK isoforms or were subjected to western blot analysis (b) for GST expression. (c) BI-D1870 inhibits RSK2-mediated p21 phosphorylation at both phospho-acceptor sites. The indicated GST-p21 wild-type (wt) and mutant proteins were incubated for the in vitro kinase assay with active RSK2 in the absence or presence of the indicated concentrations of BI-D1870 or DMSO. (d) p21 is phosphorylated by RSK2 at serines 116 and 146. The Coomassie-stained gel (upper panel) shows that approximately equal amounts of the indicated full-length GST-p21 wild-type and mutant proteins were incubated for the in vitro kinase assays with active RSK2 (middle panel) or active Akt1 (lower panel). Autophosphorylated RSK2 and Akt1 are indicated. (e) p21 is also phosphorylated by RSK1 and RSK3 at serines 116 and 146. Equal amounts of the indicated GST-p21 wild-type and mutant proteins were incubated for the in vitro kinase assays with active RSK1 (upper panel) or active RSK3 (lower panel). Autophosphorylated RSK1 and RSK3 are indicated

immunoprecipitated from MCF-7/casp3 and HCT116 cells (not shown), indicating RSK participation in this event. Ser116 phosphorylation, however, was not verified by MALDI-MS (not shown). As also our attempt to generate a phospho-Ser116 p21 antibody was unsuccessful, it is presently unclear whether p21 is phosphorylated in vivo exclusively at Ser146 upon PMA stimulation.

Next, we examined p21 expression in HCT116 cells following siRNA-mediated depletion of one or more RSKs. Whereas western blot analyses demonstrated knockdowns of RSK1 and RSK2 in both wild-type and p53-deficient HCT116 cells, because of the lack of reliable RSK3 antibodies realtime PCR analyses were required to assess the successful suppressive effect of RSK3 siRNA (Figures 6c-f). Surprisingly, however, neither individual RSK knockdowns nor their combined depletion resulted in p21 upregulation or in senescence induction (Figures $6 \mathrm{c}$ and $\mathrm{d}$; not shown). Moreover, SL0101, another potent RSK inhibitor, ${ }^{14,15}$ was also incapable of upregulating p21 expression and inducing cellular senescence in both wild-type and p53-deficient HCT116 cells (Figure 7a; Supplementary Figure S3). In addition, whereas BI-D1870 rescued only HCT116/p53 - / cells from $\gamma / R$-induced apoptosis and by itself induced massive apoptosis in p21-deficient cells, SL0101 displayed no apoptotic potential and protected both checkpoint-deficient cell lines in a p21-independent manner, indicating different target usage (Figures $7 \mathrm{~b}$ and $\mathrm{c}$ ).

As these results strongly argued against our initial hypothesis that BI-D1870 induces p21 accumulation and senescence via RSK inhibition, we finally utilized two different approaches to analyze whether the fate of p21 is altered in vivo by activated RSKs. First, we compared the half-life of exogenously expressed Flag-p21wt in MCF-7/casp3 cells in the absence and presence of PMA. Although PMA induced phosphorylation and activation of RSK1/2/3, Flag-21wt levels declined with comparable rates regardless of whether or not 

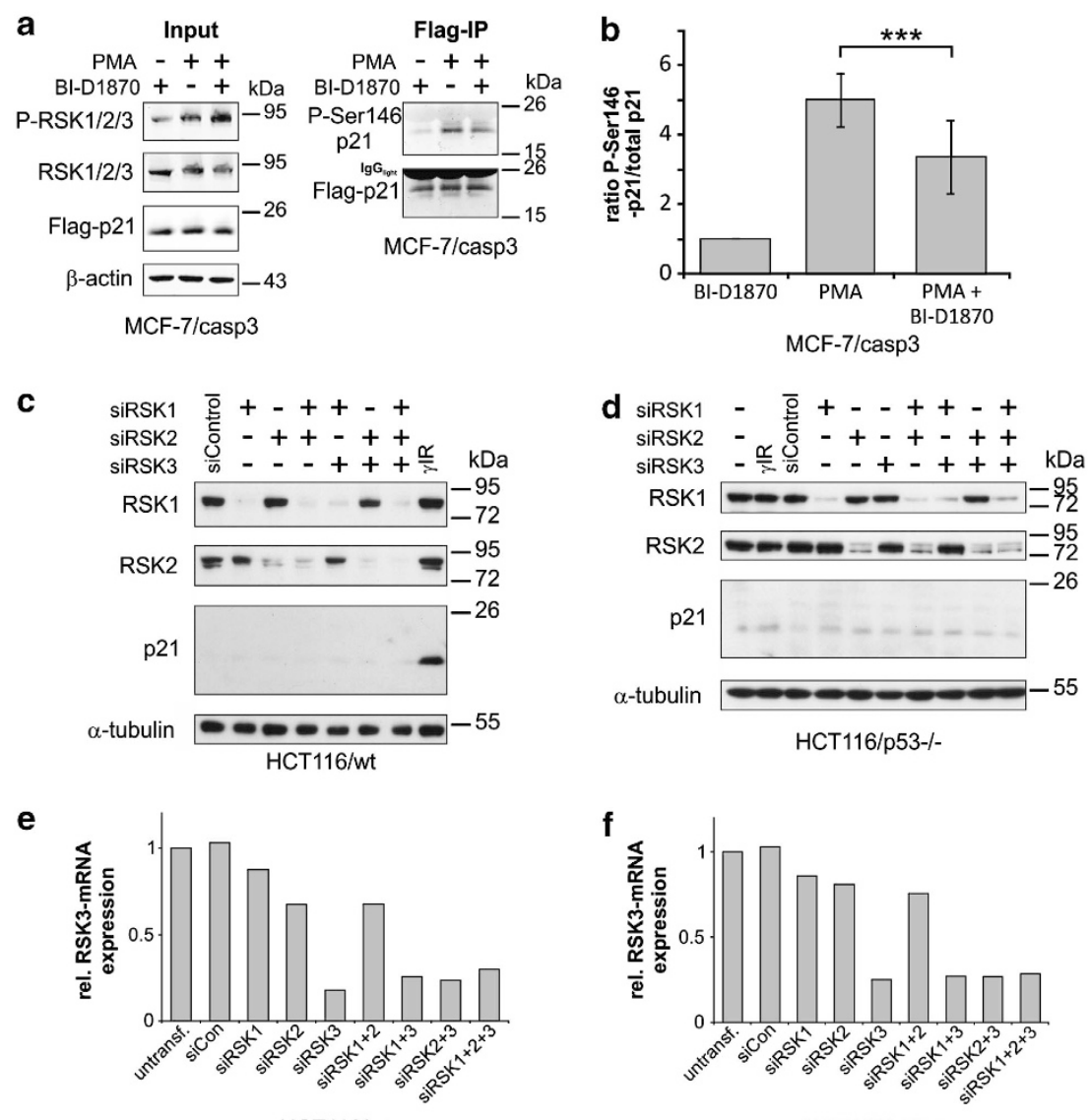

HCT116/wt

HCT116/p53-/-

Figure 6 Knockdown of RSKs does not induce p21 expression. (a and b) PMA induces in vivo p21 phosphorylation at Ser146. $24 \mathrm{~h}$ after transfection with a Flag-p21 wildtype construct; MCF-7/casp3 cells were treated for $1 \mathrm{~h}$ as indicated (PMA, $10 \mathrm{ng} / \mathrm{ml} ; \mathrm{BI}-\mathrm{D} 1870,10 \mu \mathrm{M})$ in the presence of MG-132 $(10 \mu \mathrm{M})$ before they were harvested and the cellular extracts subjected to immunoprecipitation with Flag-agarose. Both, the cellular extracts (Input) and the precipitates, (Flag-IP) were analyzed by western blotting. One representative experiment out of six is shown. (b) Densitometric analysis of the amount of phosphorylated Ser146-p21 relative to the total precipitated p21 protein. Shown is the mean + I - S.D. ( ${ }^{* \star *} P<0.005$, paired Student's $t$-test) of six independent experiments performed as described in (a) and normalized to the amount detected in BI-D1870 treated cells. Three experiments were analyzed using scanned ECL-Western Blots and the ImageJ software, whereas three other experiments were analyzed using the LI-COR infrared imaging system and Odyssey software. (c and d) Western blot analysis for the status of RSK1, RSK2 and p21 $48 \mathrm{~h}$ (c) and $72 \mathrm{~h}$ (d) after the indicated cell lines were transfected with a control siRNA or with siRNAs targeting RSK1, RSK2 or RSK3. One sample containing gamma-irradiated cells was also included in c. One representative experiment out of three is shown. (e and $\mathbf{f})$ Due to the lack of a reliable RSK3 antibody, we assessed the effect of the RSK3 siRNA by real-time PCR analyses. mRNA was isolated and analyzed with a specific Taqman probe for expression of the RSK3 mRNA. The $\beta$-actin probe was used as a normalization control. One representative experiment out of two is shown

the cells were exposed to PMA (Figure 7d). Similar results were obtained in HCT116 cells and when $\gamma \mathrm{IR}$-induced endogenous p21 levels were monitored in both cell lines following their treatment with $\mathrm{Chx}$ in the absence or presence of PMA (not shown).

As PMA can also activate other kinases including protein kinase $C(P K C)$ isoforms that are known to phosphorylate p21, thereby altering its stability, ${ }^{20}$ we employed h-TERTimmortalized retinal pigment epithelial (RPE1) cells that upon doxycyclin treatment overexpress a constitutively active FlagRSK2-CA enzyme. ${ }^{9}$ Following their exposure to $\gamma \mathrm{IR}$, expression of endogenous p21 was monitored over a period of $10 \mathrm{~h}$ in the absence and presence of doxycyclin. However, the massive doxycycline-induced expression of Flag-RSK2-CA had no effect on $\gamma \mathrm{IR}$-induced p21 levels (Figure 7e). Moreover, as also the Chx-mediated decline of exogenously expressed Flag-p21wt proceeded with similar kinetics in the absence and presence of doxycyclin-activated Flag-RSK2-
CA (not shown), these data strongly argue against our hypothesis that p21 becomes degraded following phosphorylation by RSKs. Consistently, whereas several proteins known to interact with p21, including cyclins, CDKs and peripheral cell nuclear antigen (PCNA), were clearly detectable in anti-Flag precipitates of MCF-7/casp3 cells exogenously expressing Flag-p21wt, total or activated (phosphorylated) RSKs were conspicuously absent (Supplementary Figure S4). This suggests that BI-D1870 mediates the observed effects (p21 accumulation, apoptosis modulation, senescence induction) independently of RSK inhibition.

\section{Discussion}

Mutational activation of RAS proteins is associated with the occurrence of almost $30 \%$ of all human tumors, but due to offtarget and severe side effects clinical trials based on small 
a

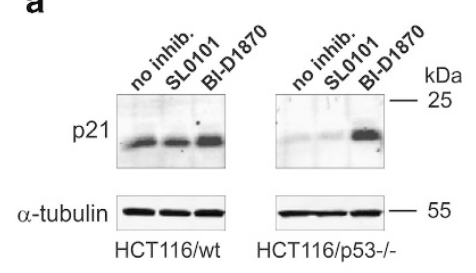

b

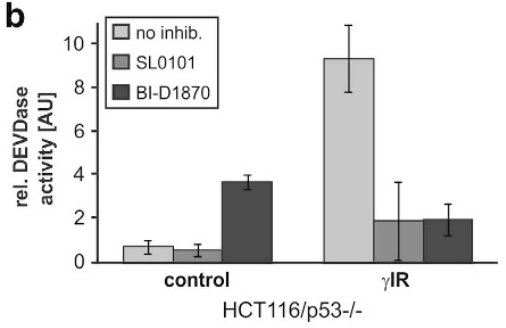

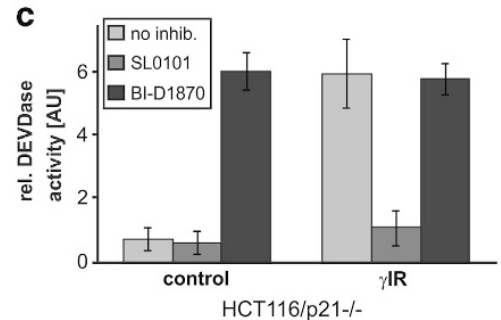

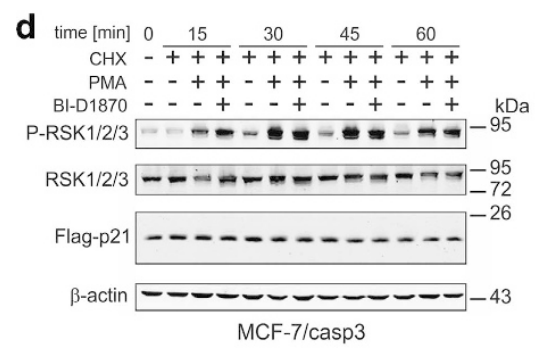

e

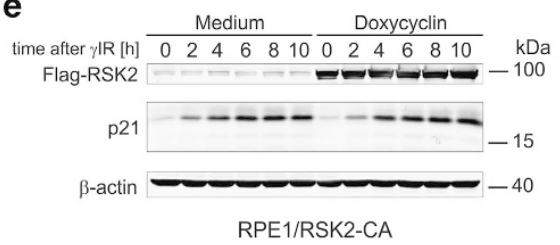

MCF-7/casp3

Figure 7 Effects of SL0101 and active RSKs on p21 expression and apoptosis. (a) HCT116 wild-type and p53 - / - cells were either left untreated or treated for $24 \mathrm{~h}$ with SL0101 $(100 \mu \mathrm{M})$ or BI-D1870 $(10 \mu \mathrm{M})$ before their cellular extracts were analyzed by western blotting for p21 expression. One representative experiment out of four is shown. (b and c) The indicated checkpoint-deficient HCT116 cells were pre-incubated for $2 \mathrm{~h}$ with SL0101 (100 $\mu \mathrm{M})$ or BI-D1870 (10 $\mu \mathrm{M})$ before they were gamma-irradiated. After 3 days, cells were analyzed for DEVDase-like activities. Shown is the mean + I - S.D. of 4-10 independent experiments. (d) MCF-7/casp3 cells were transfected with Flag-p21wt $24 \mathrm{~h}$ prior to treatment with PMA (10 ng/ml), CHX $(10 \mu \mathrm{g} / \mathrm{ml})$ and BI-D1870 $(10 \mu \mathrm{M})$. After the indicated time points cellular extracts were analyzed by western blotting for the status of active P-RSK1/2/3, total RSK1/2/3 and Flag-p21. One representative experiment out of three is shown. (e) RPE1 cells that upon doxycyclin treatment express a constitutively active RSK2 mutant (Flag-RSK2-CA) were cultured for $16 \mathrm{~h}$ in the absence or presence of doxycyclin ( $100 \mathrm{ng} / \mathrm{ml})$ before they were gamma-irradiated. At the indicated time points post irradiation, cells were harvested and cellular extracts were analyzed by western blotting for the status of Flag-RSK2-CA and endogenous p21. One representative experiment out of two is shown

molecule inhibitors targeting participating downstream kinases including Raf, MEK and ERK1/2 have so far not been very successful. ${ }^{34}$ As a consequence, further efforts are required to identify additional targets that control fewer downstream pathways and that are thus less likely to mediate such severe side effects upon their inhibition. RSKs might represent such targets, as they are almost exclusively activated downstream of ERK1/2, controlling the proliferation and survival of a variety of cancer cell lines. ${ }^{1,35}$ Particularly, RSK2 was shown to act as a key regulator in cellular transformation and metastasis. ${ }^{36-38}$ Thus, several pre-clinical studies point to RSKs as potential therapeutic targets for various cancer types, including breast, prostate and multiple myeloma. ${ }^{14,15,34,38-43}$ However, such an intervention requires detailed knowledge about the in vivo effects of RSK inhibitors. $^{34,44}$

Together with SL0101 ${ }^{15}$ and FMK, ${ }^{43}$ the dihydropteridione BI-D1870 was among the first molecules identified inhibiting all four RSK isoforms in vitro and in vivo with a >500-fold greater selectivity over many other kinases tested, including 10 closely related AGC kinases. ${ }^{29}$ As BI-D1870 appears to be slightly more potent than SL0101, ${ }^{30}$ and because FMK inhibits the C-terminal kinase domain and thus does not affect RSKs activated by a CTKD-independent mechanism, ${ }^{45}$ we decided to use BI-D1870 in our study. In various BI-D1870-treated cell lines we observed a massive transcription- and p53-independent accumulation of $\mathrm{p} 21$, which, in line with its crucial role as a cell fate modulator, ${ }^{19,23,28}$ induced p21-driven senescence and protected p21-proficient cells from $\gamma \mathrm{IR}$-induced apoptosis. On the other hand, BI-D1870 was unable to mediate these effects in p21-deficient cells, which in contrast were efficiently killed by this compound even in the absence of irradiation. As BI-D1870 also induced apoptosis in p21-proficient cells, albeit to a much lesser extent, our data suggest that this compound bears an important anti-tumorigenic potential that, however, appears to be efficiently counteracted by its own capability to upregulate the expression of anti-apoptotic p21. ${ }^{19}$

As BI-D1870 treatment resulted specifically in p21 upregulation without affecting the expression of other CDK inhibitors such as p27 and p57, we assumed a close functional relationship between RSKs and p21. Indeed, in vitro kinase assays identified p21 as a novel RSK substrate, and thus we hypothesized that in vivo p21 becomes degraded following phosphorylation by RSKs. To our surprise, however, we accumulated several lines of evidence that clearly argue against this hypothesis. First, co-immunoprecipitation studies failed to reveal an interaction between RSKs and exogenously expressed Flag-p21wt. Second, neither PMA-induced RSKs nor the more specific doxycycline-mediated induction of constitutively active RSK2-CA ${ }^{9}$ altered irradiation-induced endogenous p21 levels or those of an exogenously expressed Flag-p21wt protein. Third, comparison of the cellular effects mediated by either BI-D1870 or SL0101, another specific RSK inhibitor, ${ }^{15}$ revealed that both compounds most likely target different signaling pathways. Although SL0101 completely failed to increase p21 levels or to drive cells into senescence, both inhibitors prevented $\gamma \mathrm{IR}$-induced apoptosis albeit via p21-independent and p21-dependent pathways, respectively. As also senescence induced by U0126 proceeded in a p21independent manner, these findings further argue against our initial hypothesis that BI-D1870 mediates the observed cellular stress responses via RSK inhibition. Finally, and most importantly, siRNA-mediated depletion of individual RSKs and even their combined loss did not induce p21 upregulation or senescence. Together, these findings provide strong 
evidence that the cellular events observed following BI-D1870 exposure are mediated via an as yet unknown pathway that, besides its known off-targets PLK1 and AuroraB, does also not involve RSKs.

Despite the obvious ineffectiveness of activated RSKs on the expression and stability of p21, it may still be worthwhile knowing whether RSKs target p21 in vivo. Unfortunately, we are presently unable to answer this question. MALDI-MS and mutagenesis analyses identified in vitro two RSK phosphoacceptor sites in the recombinant GST-p21 protein from which Ser146, but not Ser116, is embedded within an RSK consensus motif. It is not uncommon that kinases including AGC kinases phosphorylate their targets at atypical sites $^{8,46-48}$ and that, on the other hand, even perfect matches of recognition sequences do not guarantee in vivo phosphorylation by an appropriate kinase. ${ }^{49}$ However, MALDI-MS analyses of Flag-p21wt immunoprecipitated from PMAstimulated MCF-7/casp3 and HCT116 cells only verified in vivo phosphorylation of Ser146, but not of Ser116. Although PMA-stimulated S146-phosphorylation of Flag-p21wt was significantly prevented following co-treatment with BI-D1870, due the above-described off-target effects of this compound it is presently unclear whether RSKs indeed phosphorylate p21 also in vivo at Ser146.

Nevertheless, regardless of whether or not RSKs target p21 in vivo, according to our data, PMA-mediated p21 phosphorylation at Ser146 alters neither its expression nor its stability. This finding further adds to the controversial effects reported on Ser146-phosphorylated p21. ${ }^{20}$ Whereas phosphorylation of this residue by PKC $\delta$ increased p21 stability, ${ }^{50}$ a decrease in its half-life was observed following Ser146 phosphorylation by the atypical $\mathrm{PKC} \zeta .{ }^{49}$ Thus, cell-type specificities may determine the fate of Ser146-phosphorylated p21. Consistent with earlier reports, however, demonstrating that Ser146 does not lie within an Akt consensus motif, ${ }^{51}$ was our finding that only Thr145, but not Ser146, is phosphorylated by Akt, whereas Ser146, but not Thr145, is targeted by RSKs, at least in vitro. In fact, an alanine substitution of one of these sites appears to enhance phosphorylation of the adjacent residue, suggesting that a conformational alteration at these sites facilitates recognition by the respective kinase. Although this observation also implies that phosphorylation of these adjacent sites might only occur in a mutually exclusive manner, confirmation of this hypothesis under in vivo conditions was not within the scope of the present study and requires further investigation.

In summary, we have uncovered for the first time opposite cellular effects induced by the RSK inhibitor BI-D1870: induction of apoptosis versus induction of apoptosis resistance and senescence most likely due to the accumulation of p21. Although our data strongly suggest that BI-D1870 mediates these cellular stress responses independently of RSKs, they nevertheless warrant further pre-clinical studies aimed to decipher the signaling pathways involved.

\section{Materials and Methods}

Cell lines, reagents and antibodies. HCT116 colon carcinoma cells and their checkpoint-deficient variants (p53-/ - and p21 $-/-$ ) were maintained in McCoy's-5A medium (PromoCell; Heidelberg, Germany). MCF-7/casp3 breast carcinoma cells were cultured in RPMI 1640 (PAA Laboratories; Linz, Austria) with
$400 \mu \mathrm{g} / \mathrm{ml}$ neomycin. ${ }^{52}$ Their identities were confirmed by DNA fingerprinting (DSMZ; Braunschweig, Germany). H-TERT-immortalized retinal pigment epithelial (RPE1) cells that upon doxycyclin treatment overexpress a constitutively active (CA; Y707A) Flag-RSK2 enzyme were cultured in DMEM/F12 (1:1) (Gibco, Life Technologies, Darmstadt, Germany). ${ }^{9}$ The media were supplemented with $10 \%$ heat-inactivated fetal calf serum, $10 \mathrm{mM}$ glutamine, $100 \mathrm{U} / \mathrm{ml}$ penicillin and $0.1 \mathrm{mg} / \mathrm{ml}$ streptomycin (PAA Laboratories). The fluorometric caspase-3 substrate Ac-DEVD-AMC and MG-132 were from Biozol (Eching, Germany). The RSK inhibitors BI-D1870 and SL0101 were from Hilary McLauchlan (Dundee, Scotland, UK) and Toronto Research Chemicals Inc. (North York, ON, Canada), respectively. The MAP kinase inhibitors U0126, SB203580 and the JNK inhibitor-I (L)-Form, as well as the p53 monoclonal DO-1 antibody, were purchased from Calbiochem (Bad Soden, Germany). The p21 and p27 monoclonal antibodies were from BD Biosciences (Heidelberg, Germany). From Cell Signaling Technology (Danvers, MA, USA) were the polyclonal rabbit antibodies recognizing AuroraB, p57, RSK1 and RSK2, and the monoclonal rabbit antibodies against all three RSK isoforms (RSK1/2/3) and PLK1. From Neomarkers (Fremont, CA, USA) were the antibodies against cyclin D1 and D3. The polyclonal rabbit antibody toward activated phospho-RSK1/2/3 (Ser380) was from R\&D Systems (Wiesbaden, Germany). Monoclonal PCNA and the polyclonal CDK2 and P-Ser146 p21 antibodies were from Santa Cruz Biotechnologies (Heidelberg, Germany). From Sigma-Aldrich (Deisenhofen, Germany) we obtained the $\beta$-actin $\mathrm{mAb}$ (AC-74), $\alpha$-tubulin mAb, the polyclonal Flag antibody, X-Gal, cycloheximide (Chx), PMA (phorbol-12-myristate-13-acetate), doxycyclin, the protease inhibitors PMSF, aprotinin, leupeptin and pepstatin and the phosphatase inhibitors sodium orthovanadate and sodium pyrophosphate.

Recombinant proteins, site-directed mutagenesis and kinase assays. The following human recombinant proteins were used: His-RSK1 and GST-Akt1 were from Millipore (Schwalbach, Germany) and Cell Signaling Technology (Danvers, MA, USA), respectively, and the GST-tagged RSK2 and RSK3 kinases were from Biozol. GST-p21 was from Calbiochem. The plasmid encoding the GST-tagged wild-type p21 protein was a kind gift from Mien-Chie Hung (Houston, TX, USA). To generate GST-p21 defective in potential acceptor sites for RSK-/Akt-dependent phosphorylation, serines and threonines were changed to alanines by site-directed mutagenesis using the QuikChange Kit according to the manufacturer's instructions (Agilent Technologies; Santa Clara, $\mathrm{CA}$, USA). Kinase reactions were carried out for $30 \mathrm{~min}$ at $30^{\circ} \mathrm{C}$ in $5 \mathrm{mmol} / \mathrm{MOPS}$ (pH 7.2), $5 \mathrm{mmol} / / \mathrm{MgCl}_{2}, 1 \mathrm{mmol} / /$ EGTA, $0.4 \mathrm{mmol} / / \mathrm{EDTA}, 2.5 \mathrm{mmol} / \mathrm{l}$ $\beta$-glycerophosphate, $50 \mu \mathrm{mol} / / \mathrm{DTT}, 50 \mu \mathrm{mol} / / \mathrm{ATP}$ and $5 \mu \mathrm{Ci}\left[\gamma^{32} \mathrm{P}\right] A T P$. The reactions were stopped by adding $5 x$ SDS sample buffer and heating $\left(5 \mathrm{~min}\right.$ at $\left.95^{\circ} \mathrm{C}\right)$. Following separation on SDS polyacrylamide gels, the gels were fixed in $50 \%$ methanol and $10 \%$ acetic acid, dried and exposed to X-ray film.

Treatment of cells, assessment of senescence-associated $\beta$-galactosidase (SA- $\beta$-Gal) activity and flow cytometric analyses. Cells were exposed to $\gamma \mathrm{IR}$ (20 Gy at $200 \mathrm{kV}$ ) using a Gulmay RS225 X-ray system from IsodoseControl (Bochum, Germany) or to the various kinase inhibitors for the indicated times. Staining for $\mathrm{SA}-\beta$-Gal activity was performed as described. ${ }^{28}$ Pictures were taken on an Axio ObserverA1 microscope using the corresponding AxioVision Software (Carl Zeiss; Göttingen, Germany). For flow cytometric analysis, cells were trypsinized, washed in PBS and resuspended in $0.1 \%$ sodium citrate, $0.1 \%$ Triton $\mathrm{X}-100$ and $50 \mu \mathrm{g} / \mathrm{ml}$ propidium iodide. After $30 \mathrm{~min}$ on ice in the dark, cells were analyzed on a FACS Calibur flow cytometer (Becton Dickinson; Heidelberg, Germany). The software CellQuestPro was used to categorize cell cycle distribution based on the relative DNA content.

Immunoprecipitation, cell extracts and western blotting. Extracts of cells transfected with a Flag-p21wt construct (Addgene; Cambridge, MA, USA; $\# 16240$ ) were normalized to equal protein concentrations and volume before they were incubated together with $30 \mu \mathrm{l}$ Flag-agarose at $4{ }^{\circ} \mathrm{C}$ overnight on a rotator. After several washing steps with lysis buffer, the precipitated proteins were analyzed by western blotting. Cell extracts were prepared in $50 \mathrm{mM}$ Tris/ $\mathrm{HCl}$ $(\mathrm{pH} 7.4), 150 \mathrm{mM} \mathrm{NaCl}, 1 \% \mathrm{NP}-40$ and protease/phosphatase inhibitors, as described. ${ }^{28}$ Protein concentrations were determined with the BioRad assay, followed by separation in SDS-polyacrylamide gels and electroblotting onto nitrocellulose membranes (Whatman; Dassel, Germany). Following antibody incubation, proteins were either visualized by enhanced chemiluminescent staining using ECL reagents (Amersham Biosciences; Braunschweig, Germany) or by the 
use of the LI-COR infrared imaging system and Odyssey software (LI-COR Biosciences, Bad Homburg, Germany).

Fluorometric determination of caspase-3-like-activity (DEVDase assay). Cell extracts measuring $50 \mu \mathrm{g}$ were incubated for $3-4 \mathrm{~h}$ with $50 \mu \mathrm{M}$ of the caspase- 3 substrate Ac-DEVD-AMC in $200 \mu$ l buffer containing $50 \mathrm{mM}$ HEPES (pH 7,4), $100 \mathrm{mM} \mathrm{NaCl}, 10 \%$ sucrose, $0.1 \%$ CHAPS and $10 \mu \mathrm{M}$ DTT. Release of fluorogenic AMC was measured at an excitation wavelength of $346 \mathrm{~nm}$ and an emission wavelength of $442 \mathrm{~nm}$ using an Infinite M200 microplate reader (Tecan, Langenfeld, Germany). The detected fluorometric signal that directly correlates to the caspase activity in cell extracts is expressed in arbitrary units (AU). A paired Student $t$-test was employed for statistical analysis.

Transfection of siRNAs and plasmids. ON-TARGETplus SMARTpool siRNAs containing four individual siRNAs were purchased from Dharmacon (Lafayette, CO, USA). The knockdown was performed according to the manufacturer's instructions. Forty-eight hours post transfection, cells were analyzed by western blotting and by fluorometric caspase assays for successful knockdowns, for the effect of these knockdowns on p21 expression and DEVDase activity. Cells were transfected with Flag-p21 using Lipofectamine 2000 according to the manufacturer's instructions (Invitrogen; Karlsruhe, Germany).

Real-time PCR. Total RNA was isolated using the RNeasy Kit (Qiagen; Hilden, Germany) according to the manufacturer's protocol. Reverse transcription was achieved with the High Capacity cDNA Kit (Applied Biosystems; Darmstadt, Germany). Taqman gene expression probes for human p21, RSK3, $\beta$-actin and GAPDH mRNA (Applied Biosystems) were employed to analyze their relative expression levels using the 7300 Real-Time PCR system (Applied Biosystems). GAPDH and $\beta$-actin mRNA served as endogenous normalization controls. The fold induction of the p21 or RSK3 mRNA was calculated via the $2^{\wedge}(\Delta(\Delta \mathrm{Ct}))$ method, thereby normalizing all samples to the level of the analyzed RNA in untreated control cells.

\section{Conflict of Interest}

The authors declare no conflict of interest.

Acknowledgements. We are grateful to B. Vogelstein for the HCT116 cell lines and to Mien-Chie Hung for the GST-p21 plasmid. We also thank T. Hoffmann for the GST and GST-p53 plasmids and J. Kehl and C. Disselhoff for excellent technical assistance. This work was supported by grants from the Deutsche Forschungsgemeinschaft (SFB 728 and JA-1060/3-1) to RUJ and the Forschungskommission of the Heinrich-Heine-University of Düsseldorf to DS and RUJ.

1. Anjum R, Blenis J. The RSK family of kinases: emerging roles in cellular signalling. Nat Rev Mol Cell Biol 2008; 9: 747-758.

2. Roberts PJ, Der CJ. Targeting the Raf-MEK-ERK mitogen-activated protein kinase cascade for the treatment of cancer. Oncogene 2007; 26: 3291-3310.

3. Roux PP, Blenis J. ERK and P38 MAPK-activated protein kinases: a family of protein kinases with diverse biological functions. Microbiol Mol Biol Rev 2004; 68: 320-344.

4. Xing J, Ginty DD, Greenberg ME. Coupling of the Ras-MAPK pathway to gene activation by RSK2, a growth factor-regulated CREB kinase. Science 1996; 273: 959-963.

5. Zhang L, Ma Y, Zhang J, Cheng J, Du J. A new cellular signaling mechanism for angiotensin II activation of NF-kappaB; An IkappaB-independent, RSK-mediated phosphorylation of p65. Arterioscler Thromb Vasc Biol 2005; 25: 1148-1153.

6. Shahbazian D, Roux PP, Mieulet V, Cohen MS, Raught B, Taunton J et al. The mTOR/ $\mathrm{PI} 3 \mathrm{~K}$ and MAPK pathways converge on elF4B to control its phosphorylation and activity. EMBO J 2006; 25: 2781-2791.

7. Roux PP, Shahbazian D, Vu H, Holz MK, Cohen MS, Taunton J et al. Ras/ERK signaling promotes site-specific ribosomal protein S6 phosphorylation via RSK and stimulates Cap-dependent translation. J Biol Chem 2007; 282: 14056-14064.

8. Fujita N, Sato S, Tsuruo T. Phosphorylation of p27Kip1 at threonine 198 by $p 90$ ribosomal protein S6 kinases promotes its binding to 14-3-3 and cytoplasmic localization. J Biol Chem 2003; 278: 49254-49260.

9. Li P, Goto H, Kasahara K, Matsuyama M, Wang Z, Yatabe Y et al. p90 RSK arranges Chk1 in the nucleus for monitoring of genomic integrity during cell proliferation. Mol Biol Cell 2012; 23: 1582-1592.
10. Bonni A, Brunet A, West AE, Datta SR, Takasu MA, Greenberg ME. Cell survival promoted by the Ras-MAPK signaling pathway by transcription-dependent and -independent mechanisms. Science 1999; 286: 1358-1362.

11. Dehan E, Bassermann F, Guardavaccaro D, Vasiliver-Shamis G, Cohen M, Lowes KN et al. $\beta$ TrCP- and RSK1/2-mediated degradation of BimEL inhibits apoptosis. Mol Cell 2009; 33: 109-116.

12. Peng $\mathrm{C}, \mathrm{Cho} Y \mathrm{Y}$, Zhu F, Zhang J, Wen $\mathrm{W}, \mathrm{Xu} Y$ et al. Phosphorylation of caspase-8 (Thr-263) by ribosomal S6 kinase 2 (RSK2) mediates caspase-8 ubiquitination and stability. J Biol Chem 2011; 286: 6946-6953.

13. Cho YY, He Z, Zhang Y, Choi HS, Zhu F, Choi BY et al. The p53 protein is a novel substrate of ribosomal S6 kinase 2 and a critical intermediary for ribosomal S6 kinase 2 and histone H3 interaction. Cancer Res 2005; 65: 3596-3603.

14. Clark DE, Errington TM, Smith JA, Frierson HF Jr, Weber MJ, Lannigan DA. The serine/ threonine protein kinase, p90 ribosomal S6 kinase, is an important regulator of prostate cancer cell proliferation. Cancer Res 2005; 65: 3108-3116.

15. Smith JA, Poteet-Smith CE, Xu Y, Errington TM, Hecht SM, Lannigan DA. Identification of the first specific inhibitor of p90 ribosomal 66 kinase (RSK) reveals an unexpected role for RSK in cancer cell proliferation. Cancer Res 2005; 65: 1027-1034.

16. Cho YY, Yao K, Kim HG, Kang BS, Zhen D, Bode AM et al. Ribosomal S6 kinase 2 is a key regulator in tumor promoter-induced cell transformation. Cancer Res 2007; 67: 8104-8112.

17. Shimamura A, Ballif BA, Richards SA, Blenis J. RSK1 mediates a MEK-MAP kinase cell survival signal. Curr Biol 2000; 10: 127-135.

18. Abbas T, Dutta A. p21 in cancer: intricate networks and multiple activities. Nat Rev Cancer 2009; 9: 400-414.

19. Jänicke RU, Sohn D, Essmann F, Schulze-Osthoff K. The multiple battles fought by antiapoptotic p21. Cell Cycle 2007; 6: 407-413.

20. Child ES, Mann DJ. The intricacies of p21 phosphorylation: protein/protein interactions, subcellular localization and stability. Cell Cycle 2006; 5: 1313-1319.

21. Jung YS, Qian $Y$, Chen X. Examination of the expanding pathways for the regulation of $p 21$ expression and activity. Cell Signal 2010; 22: 1003-1012.

22. Lee YS, Dutta A. MicroRNAs: small but potent oncogenes or tumor suppressors. Curr Opin Investig Drugs 2006; 7: 560-564.

23. Sohn $D$, Budach $W$, Jänicke $R U$. Caspase-2 is required for DNA damage-induced expression of the CDK inhibitor p21(WAF1/CIP1). Cell Death Differ 2011; 18: 1664-1674.

24. Zhang J, Chen X. Posttranscriptional regulation of $p 53$ and its targets by RNA-binding proteins. Curr Mol Med 2008; 8: 845-849.

25. Neise D, Sohn D, Budach W, Jänicke RU. Evidence for a differential modulation of p53-phosphorylating kinases by the cyclin-dependent kinase inhibitor p21WAF1/CIP1. Cell Cycle 2010; 9: 3575-3583.

26. Dent $P$, Yacoub A, Fisher PB, Hagan MP, Grant S. MAPK pathways in radiation responses. Oncogene 2003; 22: 5885-5896.

27. Sebolt-Leopold JS, Herrera R. Targeting the mitogen-activated protein kinase cascade to treat cancer. Nat Rev Cancer 2004; 4: 937-947.

28. Sohn D, Essmann F, Schulze-Osthoff K, Jänicke RU. p21 blocks irradiation-induced apoptosis downstream of mitochondria by inhibition of cyclin-dependent kinase-mediated caspase-9 activation. Cancer Res 2006; 66: 11254-11262.

29. Sapkota GP, Cummings L, Newell FS, Armstrong C, Bain J, Frodin M. BI-D1870 is a specific inhibitor of the p90 RSK (ribosomal S6 kinase) isoforms in vitro and in vivo. Biochem J 2007; 401: 29-38.

30. Bain J, Plater L, Elliott M, Shpiro N, Hastie CJ, McLauchlan H et al. The selectivity of protein kinase inhibitors: a further update. Biochem J 2007; 408: 297-315.

31. Lens SMA, Voest EE, Medema RH. Shared and separate functions of polo-like kinases and aurora kinases in cancer. Nat Rev Cancer 2010; 10: 825-841.

32. Pearce LR, Komander D, Alessi DR. The nuts and bolts of AGC protein kinases. Nat Rev Mol Cell Biol 2010; 11: 9-22.

33. Stevenson MA, Pollock SS, Coleman CN, Calderwood SK. X-irradiation, phorbol esters and $\mathrm{H} 2 \mathrm{O} 2$ stimulate mitogen-activated protein kinase activity in $\mathrm{NIH}-3 \mathrm{~T} 3$ cells through the formation of reactive oxygen intermediates. Cancer Res 1994; 54: 12-15.

34. Zhang J, Yang PL, Gray NS. Targeting cancer with small molecule kinase inhibitors. Nature Rev Cancer 2009; 9: 28-39.

35. Romeo Y, Roux PP. Paving the way for targeting RSK in cancer. Expert Opin Ther Targets 2011; 15: 5-9.

36. David JP, Mehic D, Bakiri L, Schilling AF, Mandic V, Priemel M et al. Essential role of RSK2 in c-Fos-dependent osteosarcoma development. J Clin Invest 2005; 115: 664-672.

37. Elf S, Blevins D, Jin L, Chung TW, Williams IR, Lee BH et al. p90RSK2 is essential for FLT3-ITDbut dispensable for BCR-ABL-induced myeloid leukemia. Blood 2011; 117: 6885-6894.

38. Kang S, Elf S, Lythgoe K, Hitosugi T, Taunton J, Zhou W et al. p90 ribosomal S6 kinase 2 promotes invasion and metastasis of human head and neck squamous cell carcinoma cells. J Clin Invest 2010; 120: 1165-1177.

39. Joel PB, Smith J, Sturgill TW, Fisher TL, Blenis J, Lannigan DA. pp90rsk1 regulates estrogen receptor-mediated transcription through phosphorylation of Ser-167. Mol Cell Biol 1998; 18: 1978-1984.

40. Sutherland RL, Musgrove EA. Cyclins and breast cancer. J Mammary Gland Biol Neoplasia 2004; 9: 95-104.

41. Stratford AL, Fry CJ, Desilets C, Davies AH, Cho YY, Li Y et al. Y-box binding protein-1 serine 102 is a downstream target of p90 ribosomal S6 kinase in basal-like breast cancer cells. Breast Cancer Res 2008; 10: R99. 
42. Stratford AL, Reipas K, Hu K, Fotovati A, Brough R, Frankum J et al. Targeting p90 ribosomal $\mathrm{S} 6$ kinase eliminates tumor-initiating cells by inactivating $\mathrm{Y}$-box binding protein-1 in triple-negative breast cancers. Stem Cells 2012; 30: 1338-1348.

43. Shimura $Y$, Kuroda J, Ri M, Nagoshi H, Yamamoto-Sugitani M et al. RSK2(Ser227) at $\mathrm{N}$-terminal kinase domain is a potential therapeutic target for multiple myeloma Mol Cancer Ther 2012; 11: 2600-2609.

44. Cohen M, Zhang C, Shokat KM, Taunton J. Structural bioinformatics-based design of selective, irreversible kinase inhibitors. Science 2005; 308: 1318-1321.

45. Zaru R, Ronkina N, Gaestel M, Arthur JSC, Watts C. The MAP kinase-activated RSK controls an acute Toll-like receptor signalling response in dendritic cells and is activated via two distinct pathways. Nat. Immunol 2007; 8: 1227-1235.

46. Boehm M, Yoshimoto T, Crook MF, Nallamshetty S, True A, Nabel GJ et al. A growth factor-dependent nuclear kinase phosphorylates p27(Kip1) and regulates cell cycle progression. EMBO J 2002; 21: 3390-3401.

47. Fujita N, Sato S, Katayama K, Tsuruo T. Akt-dependent phosphorylation of p27Kip1 promotes binding to 14-3-3 and cytoplasmic localization. J Biol Chem 2002; 277: 28706-28713.

48. Geraghty KM, Chen S, Harthill JE, Ibrahim AF, Toth R, Morrice NA et al. Regulation of multisite phosphorylation and 14-3-3 binding of AS160 in response to IGF-1, EGF, PMA and AICAR. Biochem J 2007; 407: 231-241.
49. Scott MT, Ingram A, Ball KL. PDK1-dependent activation of atypical PKC leads to degradation of the p21 tumour modifier protein. EMBO J 2002; 21: 6771-6780.

50. Oh Y-T, Chun KH, Park BD, Choi J-S, Lee SK. Regulation of cyclin-dependent kinase inhibitor p21WAF1/CIP1 by protein kinase $\mathrm{C} \delta$-mediated phosphorylation. Apoptosis 2007; 12: $1339-1347$.

51. Rössig L, Jadidi AS, Urbich C, Gadorff C, Zeiher AM, Dimmeler S. Akt-dependent phosphorylation of p21(CIP1) regulates PCNA binding and proliferation of endothelial cells. Mol Cell Biol 2001; 21: 5644-5657.

52. Jänicke RU, Sprengart ML, Wati MR, Porter AG. Caspase-3 is required for DNA fragmentation and morphological changes associated with apoptosis. J Biol Chem 1998; 273: 9357-9360.

(c) (i) () $\Theta$ Cell Death and Disease is an open-access journal published by Nature Publishing Group. This work is licensed under a Creative Commons Attribution-NonCommercialNoDerivs 3.0 Unported License. To view a copy of this license, visit http://creativecommons.org/licenses/by-nc-nd/3.0/

Supplementary Information accompanies this paper on Cell Death and Disease website (http://www.nature.com/cddis) 